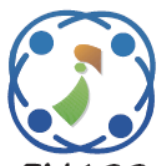

\title{
Identification and Analysis of Neurodegenerative Diseases with Twin Layered CNN Using Gait Dynamics
}

\author{
Pushpa Rani Mariathangam ${ }^{1}$ \\ Vajiha Begum Sheik Alaudin ${ }^{1 *}$ \\ Joseph Emerson Raja² \\ ${ }^{I}$ Department of Computer Science, Mother Teresa Women's University, Kodaikanal, Tamil Nadu, India \\ ${ }^{2}$ Faculty of Engineering and Technology, Multimedia University, Malaysia \\ * Corresponding author's Email: vajihabegum7391@gmail.com
}

\begin{abstract}
This study identifies the gait dynamics of Neurodegenerative disease (NDD) patients using a deep convolution neural network (CNN) approach. Human gait examination perceives individuals from the manner in which they walk, which gives clues in identifying specific disorders. Precise identification of the specific NDD assists the doctors to begin early treatment strategies. The physionet public database containing the gait dynamics of Parkinson's, Amyotrophic Lateral Sclerosis, Huntington's Diseases, and the healthy subjects is used in this work. The gait parameters such as left stride intervals, right stride intervals, left swing intervals, right swing intervals, left stance intervals and right stance intervals of each disease group are utilized. Deep Twin layered CNN (TLCNN) is applied as a feature extractor, which automatically extracts deep features from the input gait signals. Further statistical features are also measured for these inputs by implementing Fast Walsh Hadamard Transform method (FWHT). The tuned neighbourhood component analysis (TNCA) is utilized as a feature selection algorithm to select prime features from the extracted deep feature vector. The classification of each disease group is made with the selected features by the Random forest and Multi SVM machine learning approach, which gives the accuracy of $99.89 \%$, and $97.06 \%$ in classification of NDD and healthy person. Thus, the proposed TLCNN_FWHT_TNCA_RF gait classification approach based on CNN and machine learning is accurate and reliable to classify the particular neurodegenerative disorder.
\end{abstract}

Keywords: Machine learning, Convolutional neural network (CNN), Neurodegenerative diseases (NDD), Gait dynamics, Random forest.

\section{Introduction}

Neurodegenerative illnesses are depicted as problems with particular loss of neurons in the central nervous system. Neurodegenerative diseases (NDDs) weaken the brain neurons, which reflects in abnormal walking of the persons. Subsequently, examination of gait boundaries (walking pattern) is essential for a better understanding of NDDs existence. The NDD affected persons produce different abnormal gait dynamics while walking [1]. The most widely recognized brain illness is Neurodegenerative Diseases (NDD). The type of NDD is Alzheimer disease (AD), Parkinson disease (PD), Amyotrophic Lateral Sclerosis (ALS), and Huntington's disease (HD) $[2,3]$.
The brain damage or loss of dopamine in the brain can cause tremors, irregular walking, trembling of the body, and muscle rigidity [4]. The indications of AD, ALS, HD and PD are definitely not explicit with hardly noticeable nature. It makes a massive connection to one another among NDDs, prompting a misdiagnosis [5]. Neurological disorders can be diagnosed through brain imaging, gait pattern assessment, electromyogram, electrocardiogram signals analysis, etc. The recent research shows the evidence of parkinson's, Alzheimer disease diagnosis based on MRI tissue segmentation [6, 7].

The overall medical system doesn't generally keep up with exact and fast analysis. Gait analysis with AI strategies can significantly decrease diagnosis time and analysis can be accomplished in real-time. These techniques can give more precise 
findings than experienced specialists with the goal that it can achieve a supporting diagnosis of NDDs.

Numerous methodologies are being effectively applied in diagnosing NDDs, incorporating the support vector machine (SVM), random forest [3], decision tree [5], K-nearest neighbors ( $\mathrm{KNN})$, naive Bayes, neural networks [8] and long short term memory network (LSTM) classifiers [9, 10]. Furthermore, in recent years, deep learning has accomplished human competitive and sometimes better-than-human performance in solving many intellectual complications such as speech recognition and visual perception [11].

In this work person's walking pattern (gait dynamics) is analyzed for NDD diagnosis by deep learning technique. Assessing the gait dynamics gives clinical clues to identify the types of NDD. Gait analysis is a promising technology utilized by many researchers in neurodegenerative disease diagnosis, have the advantage like cost-effectiveness, with no radiation and less time-consuming. Though, it lacks accurate classification within the disease group, since the gait patterns of ALS, PD and HD are more or less similar and differ when the severity of disease increases over time and physicians need automated computerized technology that helps to classify the particular type of NDD disease precisely.

This work aims to combine the significant advantages of convolution neural network (CNN) and machine learning (ML) techniques for accurate NDD diagnosis than applying each separately. Initially, the time series gait signals of normal and NDD affected persons is taken as input, then applied proposed twin layered convolution neural network (TLCNN) model which is noise resistant and automatically extracts most prominent gait features from its fully connected layer. TLCNN model converts the $1 \mathrm{D}$ input signal into the $2 \mathrm{D}$ vector by $2 \mathrm{D}$ convolution and automatically collects the deep features and there is no need for other handcrafted feature extraction methods. Since gait signals are non-linear, the sharp discontinuities in TLCNN signal features need to accurately noted which is done by Fast Walsh Hadamard Transform (FWHT) method and statistical measures are calculated to gain absolute accuracy.

To reduce computation complexity and misclassification, feature selection is done by Tuned Neighbourhood Component Analysis (TNCA) which selects fine-tuned features for classification. The classification phase is left to machine learning (ML) algorithm to reduce the error rate and increase accuracy. Finally, classification of disease is done by ML algorithms such as random forest (RF) and Multi SVM (MSVM) with the fine-tuned features, but RF gives better results than MSVMs. Thus the combination of TLCNN and random forest (TLCNN_FWHT_TNCA_RF) offers accurate results, increases the performance, reduces the overfitting of data and computation complexity, and supports training with a minimum amount of input data than the other conventional methods. Hence, this work diagnoses and classifies NDD with a maximum accuracy rate by less computation intricacy compared to other related works and helps physicians in early diagnosis of disease by this automated computerized technology.

This paper has been organized as follows: Section 2 describes the related works in the literature. The proposed methodology is discussed in Section 3. The experimental results and discussion are described in Section 4. Finally, Section 5 gives the conclusion of this proposed work.

\section{Related works}

The different approaches for diagnosing Neurodegenerative Disorders using the gait patterns as input in the recent studies are presented.

Prabhu et al. [3] proposed a technique based on recurrence quantification analysis (RQA) to extract the non-linear gait features and achieve $96 \%$ and $100 \%$ accuracy in binary classification by SVM and PNN. This work utilizes only stride parameter as input from gait patterns which is limited data for training. Gupta et al. [5] have produced a new set of 500 gait features by implementing autocorrelation and cross-correlation functions and selecting the best features using the mutual information method. A rule-based decision tree classifier was applied to classify HD, PD, and ALS with control subjects and it yielded the classification accuracy of $88.5 \%, 92.3 \%$, $96.2 \%$, and $87.5 \%$, respectively which is a lesser accuracy rate compared to other works.

Xia et al. [13] presented the hill-climbing feature selection method in selecting Nine statistical measures from gait signals, used Leave-one-out cross-validation for evaluation, and yielded the accuracy rate $96.83 \%$, in classification of NDD. Hannink et al. [14] proposed a new method based on Deep Convolutional Neural Network (DCNN) to evaluate the stride length from the inertial sensor data. The Multidimensional Dynamic Time Wrapping method differentiated the heel-strike and Mid Stance gives $0.01 \pm 5.37 \mathrm{~cm}$ average accuracy.

Beyrami et.al. [15] presented simple method to classify the NDD based on statistical and entropy features with non-negative least square (NNNLS) classifier which gives $99.57 \%$ accuracy in control vs NDD classification. Qiang Ye et al. [8] projected 
Adaptive Neuro-Fuzzy Inference System with a neural network and Particle Swarm Optimization (PSO) algorithm to classify ND diseases. The classification of each disease group NDD, ALS, PD, HD with controls, gives an accuracy of $90.63 \%$, $93.10 \%, 90.22 \%$, and $94.44 \%$ and shows increased computation complexity.

Zhao et al. [9] proposed an LSTM model with recurrent neural network (RNN) to diagnose NDD persons and healthy persons and achieved 95.04\% accuracy with the temporal gait data. Saljuqi et al. [10] presented matching pursuit method in selecting linear and nonlinear features which combined time and frequency data of gait signal. Then applied sparse coding classifier to diagnose ALS, PD and HD gives accuracy of $93 \%, 94 \%$ and $97 \%$, which shows lesser results than other works. Gao et al. [21] demonstrated an abnormal gait recognition system based on longshort term memory (LSTM) and CNN networks. Inertial sensors collect gait data, which is then merged using the conjugate gradient technique. The local spatial characteristics are obtained using $\mathrm{CNN}$, while the temporal gait features are extracted using LSTM. The limitation in validation discovered in this strategy is due to the low amount of data and needs more data for increasing the learning rate of $\mathrm{CNN}$. This approach attained $93.1 \%$ accuracy with $6.9 \%$ error rate.

Lin et al. [23] presented a method by converting input signal into image by recurrence plot for feature extraction and applied principal neural network (PCA) for feature enhancement and finally applied $\mathrm{CNN}$ as classifier in classification of NDD vs control gives $98.91 \%$ accuracy. This work uses shorter signal data of 10 -sec from the physionet 5 min gait signal [12]. This work uses very short intervals of data, some key features might be missed which will lead to misclassification between disease groups and more training data needed for $\mathrm{CNN}$ to classify accurately and hence increases computation complexity.

Fraiwan et al. [24] proposed identification of degenerative neuromuscular disorders (DND) by various ensemble decision trees (DT) classifiers with gait signals by measuring statistical measures such as root mean square, variance, skewness, and kurtosis. Comparing with other DT models Adaptive boosting (AdaBoost) gives good result. AdaBoost model classifies normal and DND with accuracy of $99.17 \%$, $79.68 \%, 81.98 \%$ and $79.30 \%$ using VGRF data, swing, stride, stance data. In this work, noisy and redundant features reduced the learning performance of the subsequent classification models. Though, the results produced by other works is good, the relevant features produced by statistical analysis [13, 15, 24] and pattern recognition methods $[3,5,8,9,10,12$,
23 ] detects only one type of NDD disease accurately. Yet, statistical methods do not deliver reliable predictions, but pattern recognition techniques need optimal meaningful gait features for the detection system's classifier to function well.

To overcome the above limitations, computation intricacy, redundant data and noises, this work proposes a new technique with the combination of deep convolutional neural network architecture and machine learning models to diagnose all types of NDD with increased accuracy rate.

This work proposes a twin layered convolutional neural network (TLCNN) model that applies 2D convolution kernel to automatically extract the deep prominent features from the temporal gait signal data. To improve the performance of quantitative assessment, statistical features are calculated additionally with the deep features and combined as a feature vector and applied machine learning models for classification of NDD. Since more training data is needed for TLCNN to attain accurate classification, ML classifiers are used for classification, avoiding misclassification and reducing computation intricacy. For reducing noise data, feature selection is done with a combined feature vector and selecting the most contributing features among them improves accuracy and effectiveness in identifying neurodegenerative diseases.

\section{Identification of NDD with twin-layered CNN}

\subsection{NDD gait dataset}

The NDD gait dataset is collected from the Physionet public database, which measures Neuro Degenerative Diseases (NDD) severity for individual subjects based on the Vertical Ground Reaction Force (VGRF). The eight distinct sensors were set in each foot of male and female subjects and estimated the VGRF data for 15 Parkinson's disease, 19 Huntington's disease, 13 Amyotrophic lateral sclerosis (ALS) and 16 healthy control subjects. The gait signal features like stride, stance, swing, double support interval from left and right legs of one-minute recordings are presented in this database [12], which is utilized in this work. The gait dynamics are sensitively dissimilar in each disease group.

\subsection{Proposed work flow}

The proposed method is implemented by the Deep Convolution Neural Network (CNN) and machine learning (ML) techniques to identify neurodegenerative diseases (NDD). 
The block diagram of the proposed system is shown in Fig. 1. The flow of this work is listed below:

1. Stride, swing and stance time series intervals of both foot is extracted from input gait signals for each subjects.

2. Intermediate difference (ID) is made with both legs time series gait intervals for each subject.

3. Intermediate difference signal is given as input to Twin-Layered CNN (TLCNN) architecture which converts $1 \mathrm{D}$ signal to $2 \mathrm{D}$ vector by $2 \mathrm{D}$ convolution.

4. Output deep features from TLCNN is gathered and applied Fast Walsh Hadamard Transformation to calculate statistical features.

5. Then, tuned neighbourhood component analysis (TNCA) is applied for feature selection.

6. Finally, classification of NDD disease group is done by machine learning algorithms with TLCNN and statistical features.

The detailed description of each phase is explained further.

\subsection{CNN architecture}

Convolution Neural Network (CNN) is a type of feedforward neural network comprising numerous hidden layers that are commonly referred to as Deep CNN. In recent research, feature extraction and classification of ECG signals by 1D and 2D CNN models operate effectively [26]. With the succession of filters, CNN helps extract more informative deep features. The CNN model features a network including several layers, with the output of one layer coupled to the input of the next layer in a sequential manner. According to the size of the convolution filter, CNNs are classed as 1D, 2D, or 3D models. For NDD classification, the 2D-CNN model was used in this work. Convolution, pooling, and fully connected layers are the three basic interconnected layers that make up the CNN model's architecture.

\subsection{Twin layered CNN (TLCNN) technique}

The proposed TLCNN architecture consists of two convolution layers, two Rectifier Linear Unit (ReLU) layer, two normalization layers, two pooling layers, one fully connected layer and a softmax layer.

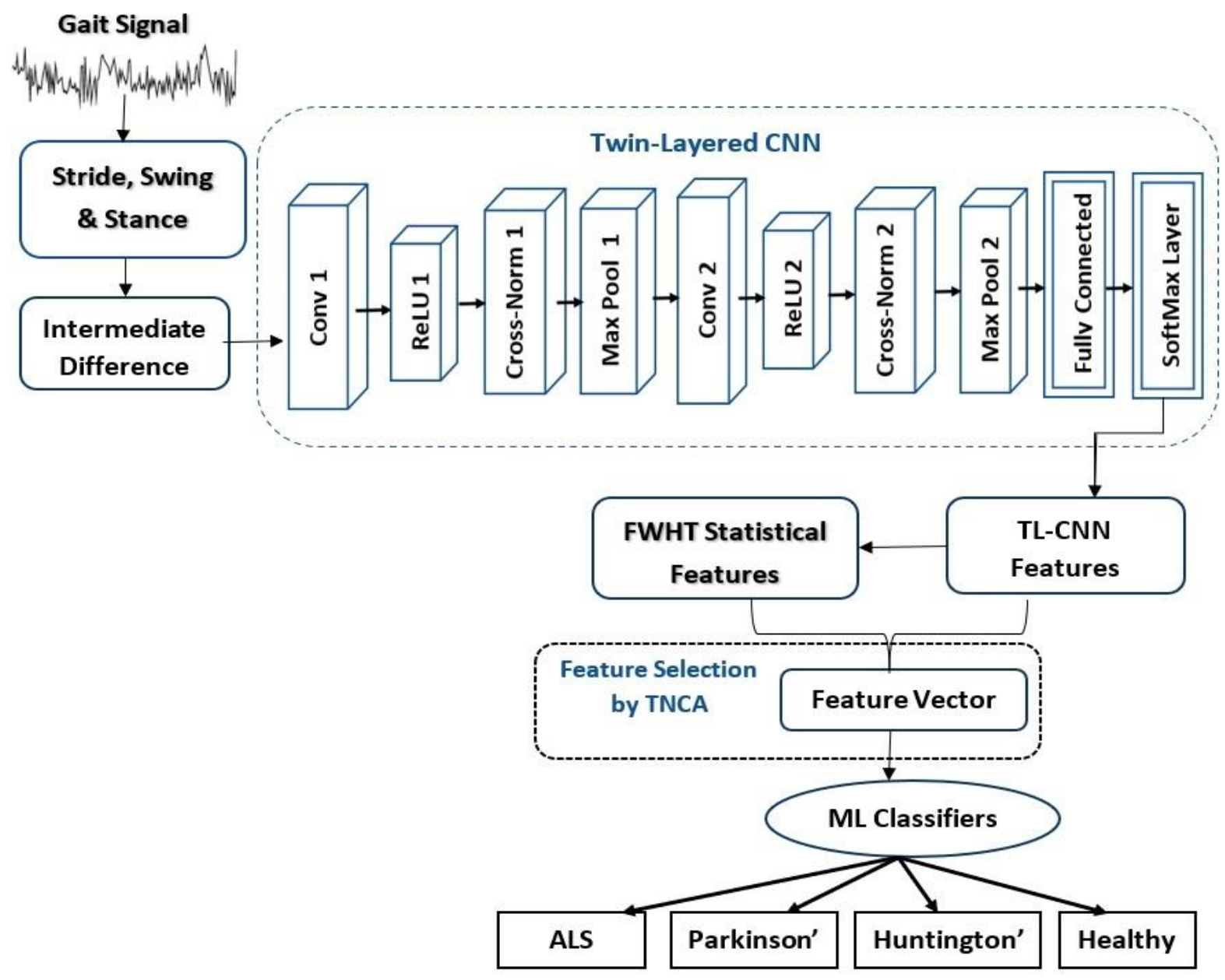

Figure. 1 Flow diagram of the proposed model 
Primarily, the input gait signals from the Physionet gait database are gathered. The gait parameters consist of left and right leg stride, swing and stance intervals are chosen. The intermediate difference (ID) of left and right leg gait signal is done as in Eq. (1), and then ID is fed as input to the Twin Layer Convolutional Neural Network (TLCNN) architecture.

$$
I D_{\mathrm{i}, \mathrm{j}}(\varepsilon)=\left(1-\left|P_{\mathrm{i}}-Q_{\mathrm{j}}\right|\right), \quad i, j=1, \ldots, N
$$

Where $P_{\mathrm{i}}$ is the left foot signal and $Q_{\mathrm{j}}$ is the right foot signal. Similarly, the intermediate difference for stride, swing and stance intervals are calculated for individual subjects.

In TLCNN, the first convolution and second convolution layer's filter size is set as $1 \times 128$ and $1 \times 64$ and the kernel is assigned as 64 and 32, respectively. The two-dimensional (2D) convolution is performed in the convolution layer (conv1) with the input intermediate difference signals of size 1x1860 and its output follows the ReLU layer. The ReLU activation function helps to maintain nonlinearity in the gait data by changing all the values from negative to zero. The normalization layer follows after the ReLU to efficiently organize the data by eliminating the redundant data and ensuring the data dependencies. Here cross-channel normalization is applied with channel size as 5, which helps perform channel-wise normalization for each element.

Next, the output of normalization layer is given as the input Max Pooling layer (pool) and then to the second convolution layer (conv2). The second convolution layer is repeated, which follows the ReLU layer, Cross-Channel Normalization layer, and Max Pooling Layer. The pooling layer helps in the reduction of parameters and enhance the computation in the CNN network. In this work, max pooling is applied, evaluating the maximum value of the feature map in each channel.

Then fully connected layer flattens the feature map from pooling layer2. The output of the fully connected layer consists of a 1024 gait dep feature vector further applied for disease classification. Tuning the CNN parameters in the training phase helps improve classification accuracy by extracting the most contributing features. Here each CNN layers kernel size, kernel value and stride number were chosen carefully. Table 1 shows the details of each layer in the proposed TLCNN architecture. Since the input data is less, CNN needs large data to train and classify accurately in the softmax layer. Hence features are extracted from it and the classification is done by a machine learning model to overcome this limitation and reduce computation difficulty.
Table 1. Layer details of the twin layered CNN architecture

\begin{tabular}{|c|c|c|c|c|}
\hline $\begin{array}{c}\text { CNN } \\
\text { Layers }\end{array}$ & $\begin{array}{c}\text { Kernel } \\
\text { Size }\end{array}$ & $\begin{array}{c}\text { Kernel } \\
\text { value }\end{array}$ & Stride & $\begin{array}{c}\text { Feature } \\
\text { Map }\end{array}$ \\
\hline Input & - & - & - & $1 \times 1860 \times 1$ \\
\hline $\begin{array}{c}\text { Conv1 } \\
+ \text { Relu1 }\end{array}$ & $1 \times 128$ & 64 & 1 & $1 \times 1860 \times 64$ \\
\hline $\begin{array}{c}\text { Cross- } \\
\text { channel } \\
\text { norm1 }\end{array}$ & - & - & - & $1 \times 1860 \times 64$ \\
\hline Pooling1 & $1 \times 2$ & - & 2 & $1 \times 930 \times 64$ \\
\hline $\begin{array}{c}\text { Conv2 } \\
+ \text { Relu 2 }\end{array}$ & $1 \times 64$ & 32 & 1 & $1 \times 930 \times 32$ \\
\hline $\begin{array}{c}\text { Cross- } \\
\text { channel } \\
\text { norm2 }\end{array}$ & - & - & - & $1 \times 930 \times 32$ \\
\hline Pooling2 & $1 \times 2$ & - & 2 & $1 \times 465 \times 32$ \\
\hline $\begin{array}{c}\text { Fully } \\
\text { connected1 }\end{array}$ & - & - & - & $1 \times 1 \times 1024$ \\
\hline \multicolumn{2}{|c|}{} & & & \\
\hline
\end{tabular}

\subsection{Statistical features}

The Fast Walsh Hadamard Transform (FWHT) method is applied with TLCNN output features to reduce complexity. An efficient FWHT algorithm which proceeds based on the divide and conquers method. FWHT method process on the size of 2 by recursive break down of Walsh Hadamard Transform (WHT) [16]. The output of FWHT is the amount of discrete WHT with the input TLCNN gait features, further used to calculate the statistical parameters such as energy, standard deviation, variance, mean and co-variance [17, 18]. The Hadamard Transform $\left(H T_{n}\right)$ works by the Eq. (2)

$$
\mathrm{HT}_{\mathrm{n}}=1 / \sqrt{2}\left(\begin{array}{cc}
\mathrm{HT}_{\mathrm{n}-1} & \mathrm{HT}_{\mathrm{n}-1} \\
\mathrm{HT}_{\mathrm{n}-1} & -\mathrm{HT}_{\mathrm{n}-1}
\end{array}\right)
$$

\subsubsection{Energy}

Energy $\left(E_{\mathrm{n}}\right)$ is measured with the FWHT features $\left(\mathrm{X}_{i}\right)$, as shown in Eq. (3). The $\mathrm{X}_{i}$ is the Hadamard transformation value of each TLCNN feature.

$$
E_{n}=\int_{-\infty}^{\infty}\left|X_{i}\right|^{2} d t
$$

\subsubsection{Standard Deviation}

The quantity of variability and reliability of each individual FWHT $\left(\mathrm{X}_{\mathrm{i}}\right)$ value is measured by the standard deviation $(\sigma)$ formula, which is presented in Eq. (4)

$$
\mathrm{SD}(\sigma)=\sqrt{\frac{\sum_{\mathrm{i}=1}^{\mathrm{S}}\left(\mathrm{X}_{\mathrm{i}}-\mu\right)^{2}}{S-1}}
$$




\subsubsection{Variance}

The square of standard deviation $(\sigma)$ value is the measure of variance $(\mathrm{Vr})$, which is shown in Eq. (5). Here, $\mathrm{s}$ is the total number of features.

$$
V r=\frac{\sum\left(\mathrm{X}_{\mathrm{i}}-\mu\right)^{2}}{S-1}
$$

\subsubsection{Mean}

The averaging of all FWHT value $\left(X_{i}\right)$ is the mean $(\mu)$ which is calculated as shown in Eq. (6).

$$
\mathrm{M}(\mu)=\frac{\sum_{\mathrm{i}=1}^{\mathrm{S}} \mathrm{X}_{i}}{S}
$$

\subsubsection{Co-Variance}

The approximation of data points in the average result of FWHT is the Co-Variance $(\mathrm{CV})$. The covariance between two data points is estimated as shown in Eq. (7).

$$
\mathrm{CV}(\mathrm{x}, \mathrm{y})=\frac{\sum_{i-1}^{S}\left(X_{i}-\bar{x}\right)\left(Y_{i}-\bar{y}\right)}{S-1}
$$

The deep TLCNN features and these five FWHT statistical features were combined as a feature vector and applied feature selection method to obtain the most contributing features for the accurate classification of diseases.

\subsection{Tuned neighbourhood component analysis (TNCA) for feature selection}

This work implements the Neighbourhood Component Analysis (NCA) [22] to choose the prime features to improve the classification accuracy and minimize the loss rate by tuning this model. NCA model works as similar to the KNN algorithm. The NCA method regulates feature weights by amplifying forecast accuracy dependent on pairwise distance and omits penalizing indicators that lead to misclassification results.

The NCA model works by enhancing the nearest neighbour classifier. The algorithm evaluates a weighting vector that corresponds to the feature vector $\left(X_{i}\right)$. The reference sample point $X_{j}$ is chosen from the samples $X_{i}$. Depending on how close the distance between two samples, the probability $P R_{i j}$ of $X_{j}$ having selected as a point of reference for $X_{i}$ from all the samples, which are higher as in Eq. (8). Where $P R_{i j}=0$,if $i=j$. The probability of selecting sample $X_{j}$ will be affected by the kernel function $\mathrm{k}$ with parameter $\sigma$ which is kernel width.

$$
P R_{i j}=\frac{\exp \left(-\left\|\mathrm{X}_{i}-\mathrm{X}_{j}\right\|^{2}\right) / \sigma}{\Sigma_{\mathrm{k}} \exp \left(-\left\|\mathrm{X}_{i}-\mathrm{X}_{j}\right\|^{2}\right) / \sigma}, P R_{i j}=0
$$

During this process, the feature weight $(W)$ are rationalized with the conjugate gradient descent by the resultant probability function as shown in $\mathrm{Eq}(9)$.

$$
\mathrm{F}(\mathrm{w})=\Sigma_{i j} p_{i j} y_{i j}-\lambda \Sigma_{r=1}^{d} W_{r}^{2}
$$

In order to avoid overfitting of parameters, $\lambda$ is introduced in Eq. (9) is known as tuned NCA (TNCA). The tuning of parameters like choosing the most acceptable value for lambda $(\lambda)$ is done based on 5-fold cross-validation on the training feature vectors. The fine-tuned $\lambda$ value is selected to minimize the loss rate for accurate classification. Our TNCA method determines the best features based on the feature weights by tuning the $\lambda$ value.

TNCA selects the optimal features from the extracted TLCNN features and FWHT statistical features. Totally 991 prominent features are chosen out of 1024 deep features and 5 statistical features by selecting the best $\lambda$ value as 0.01 for the TNCA method.

\subsection{Classification by ML algorithms}

For the classification of Neurodegenerative disease (NDD), machine learning (ML) algorithms such as Random Forest (RF), and Multi SVM classifier are utilized. The random forest classifier provides an ensemble of classification by bagging and random feature selection technique. Each tree is trained on bootstrap tests of the training data, and predictions are based on the popular votes of trees in the forest. The features are randomly selected at each node while tree growing. The RF classifier reduces the overfitting of data when multiple trees are created $[19,20]$.

SVM is a supervised two-class classification method and Multi SVM (MSVM) is a multiclass classification technique formed by multiple two-class methods. The most typical methodology in practice for multiclass classification with SVM is to develop one-to-rest classifiers and then choose the class that classifies the test dataset only with the largest margin. Another option is to create a collection of one-to-one classifiers and choose the class with the most classifiers selected. MSVM works by one to-one approach, which classifies the $n$ classes data by $n(n-1) / 2$ SVM classifiers in this proposed model.

The time required to train classifiers may be reduced because the training data set for every classifier is significantly smaller [25]. The separation hyperplane was created by Eq. (10). 


$$
f\left(X_{i}, W, b\right)=W X_{i}+b=s
$$

Where $X_{i}$ is the feature vector, $W$ is weight and $b$ is bias. Multiclass SVM implemented with RBF kernel for recognizing NDD diseases with the TNCA selected features. The prediction of disease is classified by Eq. (11).

$$
f(X)=\operatorname{argmax}_{S}\left(\Sigma_{S} f_{s, t}(X)\right)
$$

The machine learning algorithms classify NDD and healthy subjects by multiclass classification with TNCA selected features. Further binary classification such as ALS vs CO, HD vs CO, and PD vs CO is done. In this work, a random forest classifier gives the best classification result compared to Multi SVM.

\section{Results and discussion}

The proposed work observations are done using MATLAB 2019a with an intel corei3 $2.0 \mathrm{GHz}$ system. In the proposed work, physionet public database [12] is utilized to gather the gait signals of ALS, HD, PD and normal subjects. The intermediate difference between the left and right leg is taken for individual subjects and given as input to the proposed twin layered CNN (TLCNN) architecture. The stride, stance and swing gait intervals and its absolute intermediate difference of one ALS patient is represented in Fig. 2 to 4 respectively.

Filter size is manually chosen for each layer in the TLCNN architecture. Each layer in TLCNN extracts and selects the best-contributing features and finally, 1024 deep features are collected from the fully connected layer. These TLCNN deep features are further applied with the Fast Walsh Hadamard Transform (FWHT) method and some statistical features are calculated. The statistical features such as mean, variance, co-variance, energy, and standard deviation features are measured to improve accuracy.

The deep TLCNN features and five statistical features are combined as a feature vector. Then best features are selected by Tuned Neighborhood Component Analysis (TNCA) where the lambda value chosen is 0.01 by the five fold cross-validation method. These selected features are fed as input to Random Forest and Multi SVM classifiers separately to classify neurodegenerative diseases and observed each classifier's performance.

\subsection{Performance measures}

The Leave One Out Cross Validation (LOOCV) method was utilized to measure machine learning classifiers' performance in the classification of NDD.

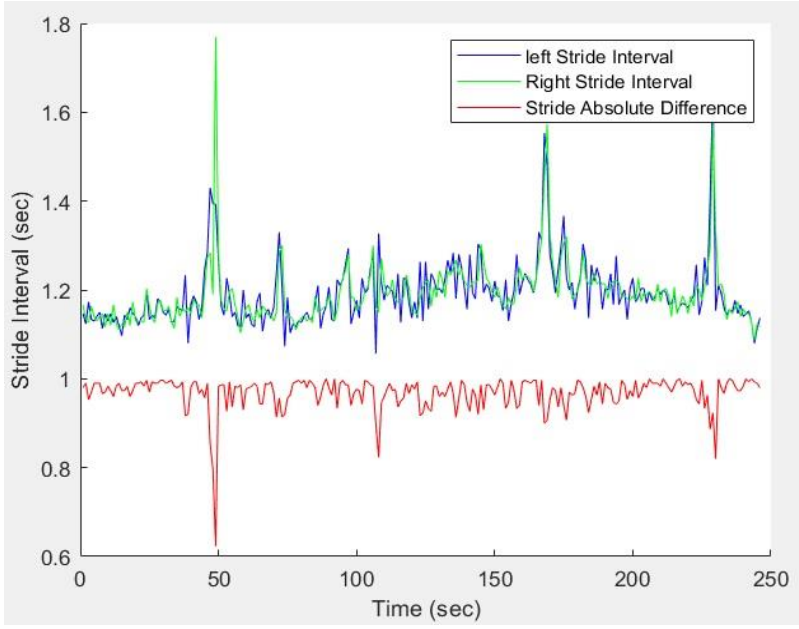

Figure. 2 Gait stride intervals and absolute difference of ALS patient

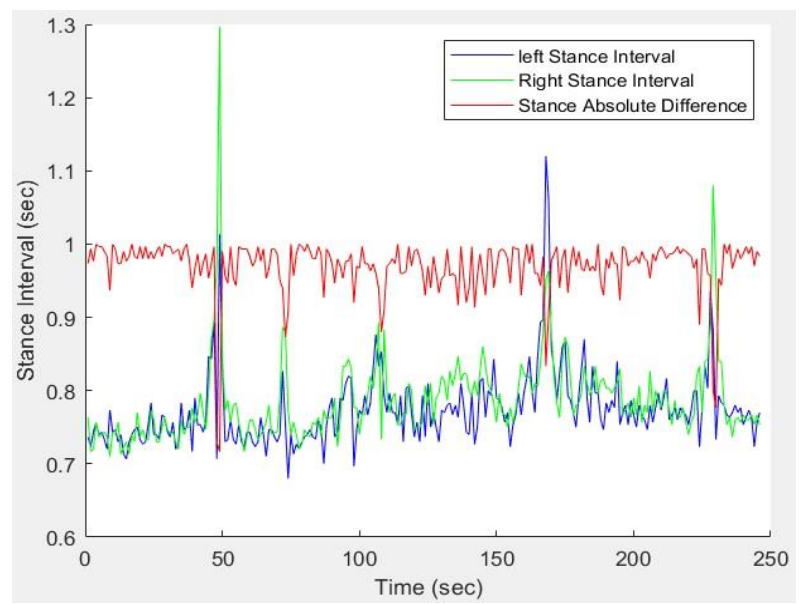

Figure. 3 Gait stance intervals and absolute difference of ALS patient

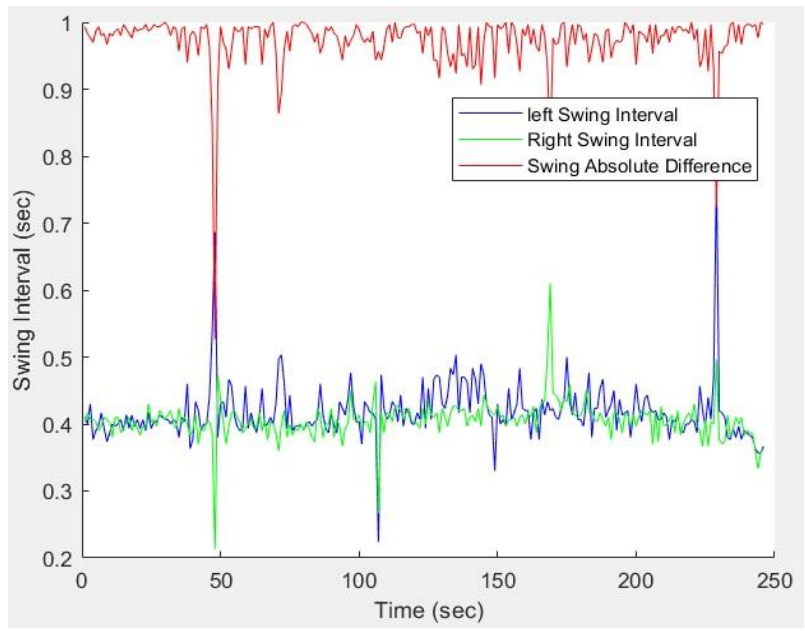

Figure. 4 Gait swing intervals and absolute difference of ALS patient

Performance measures such as accuracy (A), sensitivity $(S)$, specificity $(S P)$, and precision $(P)$ are calculated by the count of confusion matrix values, which are true positive $(T P)$, false positive $(F P)$, false 
negative $(F N)$ and true negative ( $T N)$. The performance of the proposed work is measured by the formulas below in Eq. (12) to (15):

$$
\begin{gathered}
A=\frac{T P+T N}{T P+T N+F N+F P} \\
S=\frac{T P}{T P+F N} \\
S P=\frac{T N}{T N+F P} \\
P=\frac{T P}{T P+F P}
\end{gathered}
$$

\subsection{TLCNN training method}

The training process of the TLCNN network applies adam optimizer, and the training parameters such as mini-batch size is set as 1 , max-epochs as 15 and learning rate is applied as 1e-1, 1e-2, 1e-3, 1e-4 and $1 \mathrm{e}-5$ and observed the performance of the network with each learning rate. TLCNN architecture training details on a single CPU performs 15 epochs, 47 iterations per epoch and 705 maximum iterations with each learning rate. We use adam optimizer in training options instead of stochastic gradient descent optimizer to reduce the local minimum on the error.

The learning rate is adjusted as $1 \mathrm{e}-1,1 \mathrm{e}-2,1 \mathrm{e}-3$, $1 \mathrm{e}-4$, and $1 \mathrm{e}-5$ in training with 15 epoch and extracted the deep TLCNN features for each learning rate and observed the classification performance of machine learning classifiers. The elapsed time taken during TLCNN training with each learning rate is monitored and represented in Fig. 5.

This shows only a slight variation in the timing of each learning rate during training. There is a steady rise in each learning rate's timing and shows a reduction in timing at the $700^{\text {th }}$ iteration for all learning rate's. Alteration of the learning rate

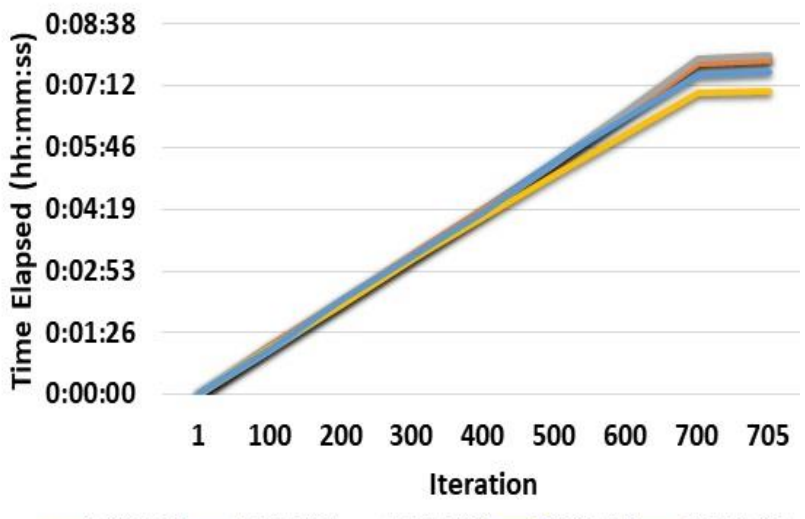

$-1.00 \mathrm{E}-01-1.00 \mathrm{E}-02-1.00 \mathrm{E}-03-1.00 \mathrm{E}-04-1.00 \mathrm{E}-05$

Figure. 5 Elapsed training time of twin layered-CNN for different learning rate does not significantly reduce the training time but impacts classification performance due to extracting optimal deep features. We observed each machine learning classifier's classification performance and timing with the features observed during the TLCNN training of each learning rate.

\subsection{Classification performance of ML classifiers}

The machine learning (ML) classifiers such as Random Forest and Multi SVM performances are estimated in terms of accuracy, sensitivity, specificity, and precision for each neurodegenerative disease (NDD) and healthy controls (CO) classification with TNCA selected features. These measures are calculated by comparing the actual test output and the predicted output by the LOOCV technique. The leave-one-out-cross-validation (LOOCV) is activated to measure the performance of the proposed system in the testing phase. For validation LOOCV method arbitrarily picks a single subject and leave the rest for the purpose of training. Here $70 \%$ of data is taken for training and the rest $30 \%$ is left for validation. For measuring the performance of the proposed work with ML classifiers, the training procedure is repeated manifold times by selecting diverse data for validation. Fig. 6 shows proposed work with ML classifiers performance in classifying NDD and normal subjects with TLCNN features collected by different learning rates.

The TLCNN features trained with 1e-3 learning rate is better and classification accuracy rate is higher than other learning rates. Hence 1e-3 learning rate is used in TLCNN architecture and these extracted features are taken for further processing in this work. Then fast walsh hadamard transformation (FWHT) is applied with the TLCNN features to calculate statistical features. Finally, TNCA method selects the best 991 features from 1024 TLCNN features and 5 FWHT statistical features.

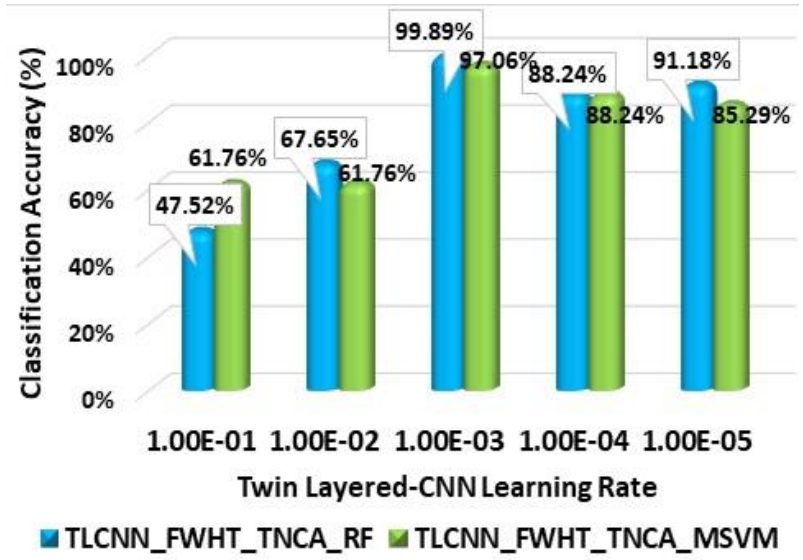

Figure. 6 Effect of different learning rate of TLCNN on classification with ML classifiers 
Table 2. Performance of the proposed models

\begin{tabular}{|c|c|c|c|}
\hline Proposed Models & Accuracy & Sensitivity & Specificity \\
\hline TLCNN & $88.24 \%$ & $75 \%$ & $90.91 \%$ \\
\hline TLCNN_FWHT_RF & $91.18 \%$ & $85.42 \%$ & $93.88 \%$ \\
\hline TLCNN_FWHT_TNCA_RF & $99.89 \%$ & $99.89 \%$ & $99.91 \%$ \\
\hline
\end{tabular}

Table 3. Performance measures of TLCNN model with ML classifiers in classification of NDD and normal

\begin{tabular}{|c|c|c|c|c|}
\hline Proposed Work & Accuracy & Sensitivity & Specificity & Precision \\
\hline TLCNN_FWHT_TNCA_RF & $99.89 \%$ & $99.89 \%$ & $99.91 \%$ & $97 \%$ \\
\hline TLCNN_FWHT_TNCA_MSVM & $97.06 \%$ & $93.75 \%$ & $97.73 \%$ & $96.43 \%$ \\
\hline
\end{tabular}

Table 4. Comparison of proposed work with related study in two groups classification (accuracy in \%)

\begin{tabular}{|c|c|c|c|c|c|c|c|}
\hline $\begin{array}{c}\text { Performance } \\
\text { Metrics }\end{array}$ & $\begin{array}{c}\text { TLCNN_FWHT } \\
\text { _TNCA_RF }\end{array}$ & $\begin{array}{c}\text { Gupta } \\
{[\mathbf{5}]}\end{array}$ & $\begin{array}{c}\text { Zhao } \\
{[\mathbf{9}]}\end{array}$ & $\begin{array}{c}\text { Beyrami } \\
{[\mathbf{1 5}]}\end{array}$ & $\begin{array}{c}\text { Lin } \\
{[\mathbf{2 3}]}\end{array}$ & $\begin{array}{c}\text { Fraiwan } \\
{[\mathbf{2 4}]}\end{array}$ & $\begin{array}{c}\text { Saljuqi } \\
{[\mathbf{1 0}]}\end{array}$ \\
\hline ALS vs CO & 98.28 & 96.2 & 97.25 & 100 & 100 & 98.8 & 94 \\
\hline HD vs CO & 100 & 88.5 & 92.25 & 99.90 & 98.41 & 99.40 & 97 \\
\hline PD vs CO & 100 & 92.3 & 96.80 & 99.78 & 100 & 98.80 & 93 \\
\hline NDD vs CO & 99.89 & 87.5 & 95.04 & 99.57 & 98.91 & 99.17 & - \\
\hline
\end{tabular}

It shows that the proposed work with Random Forest (RF) outperforms well than the Multi SVM (MSVM) in classification of NDD with the best features selected by TNCA method. The classification of neurodegenerative diseases (NDD) and healthy controls with the proposed TLCNN_FWHT_TNCA_RF technique and TLCNN_FWHT_TNCA_MSVM technique gain $99.89 \%$ and $97.06 \%$ accuracy.

The time elapsed during NDD classification by the Random Forest and Multi SVM classifiers are 135 seconds and 128 seconds with the TNCA selected features. The proposed work performance is compared by changing different $\mathrm{CNN}$ learning rates in feature extraction and found that change in learning rate and epoch, impacts much in improving classification accuracy. Table 2 presents the proposed model's performance. The first proposed model is deep twin-layered CNN (TLCNN), which automatically extracts the features and classifies the NDD and control group by the CNN SoftMax layer gives $88.24 \%$ accuracy, $75 \%$ sensitivity and $90.91 \%$ specificity. The first model increases computation intricacy and needs more data for classification by the softmax layer.

Hence, to improve the proposed models performance, the second model TLCNN_FWHT_RF is proposed. In that, FWHT is applied with TLCNN features which reduce the process complexity and measured statistical features to increase the accuracy rate. Both TLCNN and FWHT statistical features are concatenated for the classification of diseases by Random forest classifier (TLCNN_FWHT_RF), gives an improved accuracy of $91.18 \%$ compared to the TLCNN model. Finally, the third model, TLCNN_FWHT_TNCA_RF is proposed to improve the accuracy rate by implementing tuned neighbourhood component analysis (TNCA) to select the most prime features of TLCNN_FWHT feature vector and classification by random forest classifier gives $99.89 \%$ higher accuracy rate than the other two proposed models. The proposed system shows that random forest works well using the TNCA features selection method with the trained TLCNN deep features and FWHT statistical features by accurately identifying NDD and normal persons with limited input data. Table 3, presents ML classifiers (RF, MSVM) performance measures in terms of accuracy, sensitivity, specificity, and precision in classification of NDD with the TLCNN_FWHT_TNCA model. TLCNN_FWHT_TNCA_RF method gains $99.89 \%$ higher accuracy rate compared to the TLCNN_FWHT_TNCA_MSVM method, which attains $9 \overline{7} .06 \%$ accuracy.

Table 4 compares other related works and proposed work (TLCNN_FWHT_TNCA_RF) binary classification results of each two groups. It shows our proposed work gains the highest accuracy rate than the other works in literature in classification of two groups. The proposed works accuracy result of NDD vs control group (CO) gives $99.89 \%$, ALS vs CO shows $98.28 \%$ and both $\mathrm{HD}$ vs $\mathrm{CO}$ and $\mathrm{PD}$ vs $\mathrm{CO}$ gives $100 \%$ accuracy. Gupta [5] work shows lesser results than our work in disease classification with control groups such as 96.2\%, 88.5\%, 92.3\%, and $87.5 \%$ acuracy for ALS, HD, PD, and NDD. Zhao [9] work give 97.25\%, 92.25\%, 96.80, 95.04\% accuracy for ALS, HD, PD and NDD classification with controls shows lesser results than our work. Beyrami [15] and Lin [23] work shows $1.72 \%$ increased result (i.e 100\%) and Fraiwan [24] work 


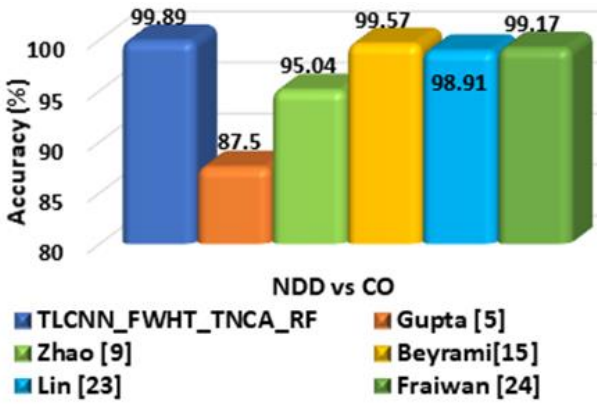

Figure. 7 Comparison of proposed work with other works in NDD vs CO group classification

shows $0.52 \%$ (i.e. $98.8 \%$ ) higher result for ALS vs $\mathrm{CO}$ than our work. Beyrami [15] work show lesser classification result for HD, PD and NDD as $99.9 \%$, $99.78 \%$ and $99.57 \%$ than our work. Lin [23] work gives $98.41 \%, 100 \%$ and $98.91 \%$ for HD, PD and NDD detection. Fraiwan [24] work showed 99.40\%, $98.80 \%$, and $99.17 \%$ accuracy for HD, PD, and NDD classification which is lesser than our proposed work. Saljuqi [10] work give 94\%, 97\%, 93\% accuracy for ALS, HD and PD which is lesser than the proposed work. The comparative result of NDD vs CO classification with related works is shown in Fig. 7.

This shows that our TLCNN_FWHT_TNCA_RF proposed work proved its efficiency in acquiring the most prominent features by TLCNN architecture, which is suited for calculating FWHT statistical features and finetuned the features by TNCA method, which minimizes the error rate. Finally, obtained the highest classification result by Random Forest (RF) classifier with best-fine-tuned features most suited for RF to automatically maintain a more accurate classification of the NDD group. This work can deal with a minimum amount of dataset with less computational intricacy than other conventional methods. In the future, this proposed automatic computerized gait technique will be combined with the person's hand movement data to enhance the system's performance.

\section{Conclusion}

This work is proposed with the combination of Deep Twin Layered Convolutional Neural Network (TLCNN) and Machine Learning algorithm to recognize and classify neurodegenerative diseases using gait dynamics. The input data of the left and right leg gait intervals of each group is taken. The CNN architecture involved in this work automatically collects the helpful features for the classification of each NDD disease and healthy controls. As a supporting parameter, the statistical features are also measured with FWHT and applied TNCA for feature selection. The efficient classification task performed with the TLCNN_FWHT_TNCA features by machine learning algorithms such as Random Forest and Multi SVM achieved an overall accuracy of $99.89 \%$ and 97.07\%, respectively. Thus, the proposed TLCNN_FWHT_TNCA_RF model proved by its performance analysis compared to the related models in the literature. Henceforth, it is possible to classify and recognize healthy persons and persons with neurodegenerative disorders using the gait signals through Deep CNN and Machine Learning methods with high accuracy and less computation intricacy. In the future, we will try to work on more real-time data and further try to enhance the system by adding hand movement data in these proposed models.

\section{Conflicts of Interest}

The authors declare no conflict of interest.

\section{Author Contributions}

All authors conceived and designed the study. The paper methodology, software, formal analysis, validation, writing-original draft preparation, writing-review and editing was carried out by Vajiha Begum Sheik Alaudin. Visualization, supervision and project administration was done by Pushpa Rani Mariathangam and Joseph Emerson Raja.

\section{References}

[1] P. Ren, S. Tang, F. Fang, L. Luo, L. Xu, M. Bringas, D. Yao, K. Kendrick, and P. V. Sosa, "Gait rhythm fluctuation analysis for neurodegenerative diseases by empirical mode decomposition", IEEE Transactions on Biomedical Engineering, Vol. 64, No. 8 pp. 5260, 2016.

[2] P. H. Chen, R. L. Wang, D. J. Liou, and J. S. Shaw, "Gait disorders in parkinson's disease: assessment and management", International Journal of Gerontology, Vol. 7, No. 4, pp. 189193, 2013.

[3] P. Prabhu, A. K. Karunakar, H. Anitha, and N. Pradhan, "Classification of gait signals into different neurodegenerative diseases using statistical analysis and recurrence quantification analysis", Pattern Recognition Letters, Vol. 139, pp. 10-16, 2020.

[4] J. M. Hausdorff, A. Lertratanakul, M. E. Cudkowicz, A. L. Peterson, D. Kaliton, and A. L. Goldberger, "Dynamic markers of altered gait rhythm in amyotrophic lateral sclerosis", Journal of Applied Physiology, Vol. 88, No. 6, pp. 2045-2053, 2000. 
[5] K. Gupta, A. Khajuria, N. Chatterjee, P. Joshi, and D. Joshi, "Rule based classification of neurodegenerative diseases using data driven gait features", Health and Technology, 2018.

[6] Y. Setiawan, C. Fatichah, and R. Sarno, "A Ne w Local Gaussian Variational Level Set for Glo bus Pallidus Segmentation", International Jour nal of Intelligent Engineering and Systems, Vol. 13, No. 5, pp. 317-326, 2020.

[7] H. S. Suresha and S. S. Parthasarathy, "Diagnosis of Alzheimer Disease Using Fast Independent Component Analysis and Otsu Multi-level Thresholding", International Journal of Intelligent Engineering and Systems, Vol. 11, No. 5, pp. 74-83, 2018.

[8] Q. Ye, Y. Xia, and Z. Yao, "Classification of Gait Patterns in Patients with Neurodegenerative Disease using Adaptive Neuro-Fuzzy Inference System", Hindawi, Computational and Mathematical Methods in Medicine, pp.1-8, 2018.

[9] A. Zhao, L. Qi, J. Li, J. Dong, and H. Yu, "LSTM for Diagnosis of Neurodegenerative Diseases using Gait Data", In: Proc. of Ninth International Conf. On Graphic and Image Processing, Qingdao, China, Vol.10615, 2018.

[10] M. Saljuqi and P. Ghaderyan, "A novel method based on matching pursuit decomposition of gait signals for Parkinson's disease, Amyotrophic lateral sclerosis and Huntington's disease detection", Neuroscience Letters, Vol. 761, No. 136107, 2021.

[11] C. Zhang, W. Liu, H. Ma, and H. Fu, "Siamese Neural Network Based Gait Recognition for Human Identification," In: Proc. of 2016 IEEE International Conf. on Acoustics, Speech and Signal Processing (ICASSP), Shanghai, China, pp. 2832-2836, 2016.

[12] www.physionet.org/physiobank/database/gaitp $\mathrm{db} /$

[13] Y. Xia, Q. Gao, and Q. Ye, "Classification of Gait Rhythm Signals between Patients with Neurodegenerative Diseases and Normal Subjects: Experiments with Statistical Features and Different Classification Models", Biomed. Signal Process. Control, No. 18, pp. 254-262, 2015. doi:10.1016/j.bspc.2015.02.002.

[14] J. Hannink, T. Kautz, C. F. Pasluosta, J. Barth, S. Schulein, K. Gabmann, J. Klucken, and B. M. Eskofier, "Mobile Stride Length Estimation with Deep Convolutional Neural Networks", IEEE Journal of Biomedical And Health Informatics, Vol. 22, No. 2, pp. 354-362, 2018.

[15] S. M. G. Beyrami and P. Ghaderyan, "A robust, cost-effective and non-invasive computer-aided method for diagnosis three types of neurodegenerative diseases with gait signal analysis", Measurement, Vol. 156, No. 107579, 2020.

[16] Fino and Algazi, "Unified Matrix Treatment of the Fast Walsh-Hadamard Transform", IEEE Transactions on Computers. Vol. 25, No. 11, pp. 1142-1146, 1976.

[17] S. A. V. Begum and M. P. Rani, "Classification of Brain Disorder using Gait Patterns", International Journal of Scientific Research in Computer Science Applications and Management Studies, Vol. 8, No. 1, 2019.

[18] S. A. V. Begum and M. P. Rani, "Recognition of neurodegenerative diseases with gait patterns using double feature extraction methods", In: Proc. of 4th International Conf. On Intelligent Computing and Control Systems (ICICCS), Madurai, India, pp. 332-338, 2020.

[19] A. Liaw and M. Wiener, "Classification and Regression by Random Forest", R. News, Vol. 2/3, pp. 18-22, 2002.

[20] L. Breiman, Random Forests, Machine Learning, Vol. 45, pp. 5-32, 2001.

[21] J. Gao, P. Gu, Q. Ren, J. Zhang, and X. Song, "Abnormal Gait Recognition Algorithm Based on LSTM-CNN Fusion Network", IEEE Access, Vol. 7, pp. 163180-163190, 2019.

[22] J. Goldberger, G. E. Hinton, S. Roweis, and R. R. Salakhutdinov, "Neighbourhood Components Analysis", In: Proc. Advances in Neural Information Processing Systems, pp. 513-520, 2004.

[23] C. W. Lin, T. C. Wen, and F. Setiawan, "Evaluation of Vertical Ground Reaction Forces Pattern Visualization in Neurodegenerative Diseases Identification Using Deep Learning and Recurrence Plot Image Feature Extraction", Sensors, Vol. 20, No. 14:3857, pp. 1-22, 2020.

[24] L. Fraiwan and O. Hassanin, "Computer-aided identification of degenerative neuromuscular diseases based on gait dynamics and ensemble decision tree classifiers", Plos One, Vol. 16, No. 6, 2021.

[25] M. Pushparani and A. Athisakthi, "Detection of Movement Disorders Using Multi SVM", Global Journal of Computer Science and Technology Interdisciplinary, Vol. 13, No. 1, 2013.

[26] A. Ullah, S. U. Rehman, S. Tu, R. M. Mehmood, Fawad, and M. Ehatisham-ul-haq, "A Hybrid Deep CNN Model for Abnormal Arrhythmia Detection Based on Cardiac ECG Signal", Sensors, Vol. 21, No. 3: 951, 2021. 